\title{
LA CORRUPCIÓN COMO RESPONSABILIDAD COMPARTIDA UNA REVISIÓN DE LAS ESTRATEGIAS ANTICORRUPCIÓN EN EL SECTOR PRIVADO
}

\author{
Pablo Biderbost \\ Universidad Pontificia Comillas, España \\ pbiderbost@comillas.edu \\ GuILleRmo BoscÁN \\ Universidad de Salamanca, España \\ boscanca@upsa.es \\ NaLia Rochin \\ BB\&R, España \\ Universidad Autónoma de Baja California, México \\ nrochin@bbyr.com
}

\section{RESUMEN}

En la actualidad, las empresas se enfrentan a mayores exigencias en materia anticorrupción. Ante la pregunta con qué instrumentos cuentan las empresas para adaptarse a estos cambios sociológicos y normativos, resulta oportuno identificar las herramientas que el sector privado puede utilizar para evitar caer en conductas inapropiadas, que minen su reputación corporativa y afecten su volumen de negocios. Ya que la lucha contra la corrupción debe ser un trabajo conjunto entre ciudadanía, sector público y privado, este artículo analiza algunas estrategias anticorrupción disponibles para las empresas. En específico, se presta especial atención a un mecanismo de planeación estratégica, conocido como generación de mapas de riesgo de corrupción.

Palabras clave: Corrupción, Instrumentos anticorrupción, Mapas de riesgo, Sector privado. 


\title{
CORRUPTION AS SHARED RESPONSIBILITY \\ A REVIEW OF ANTI-CORRUPTION STRATEGIES IN THE \\ PRIVATE SECTOR
}

\begin{abstract}
Businesses currently face increased demands as regards anti-corruption. In response to the question of the instruments that companies have to adapt to these sociological and regulatory changes, it is opportune to identify the tools which the private sector can use to avoid falling into inappropriate conduct that undermines their corporate reputation and affects their turnover. Given that citizens and the public and private sectors must work together to fight corruption, this article analyzes some of the anticorruption strategies available to companies. Specifically, it looks closely at a strategic planning mechanism known as corruption risk mapping.
\end{abstract}

Keywords: Corruption, Anti-corruption instruments, Risk maps, Private sector. 


\section{INTRODUCCIÓN}

Sin duda, la lucha contra la corrupción no es responsabilidad exclusiva de las instituciones y servidores gubernamentales. Es claro que los actos ilícitos de desvío de recursos públicos requieren, ineludiblemente, de la participación de ciudadanos y empresas privadas. Como respuesta, se aprecia la tendencia en años recientes de creación de departamentos de compliance al interior de las empresas.

A ello, han conducido presiones de dos tipos. Por un lado, desde el punto de vista sociológico, un buen número de ciudadanos organizados que a través de diferentes mecanismos exigen altos estándares éticos tanto al sector público como al sector privado. Por otro lado, la legislación internacional y nacional obliga a la asunción de estos estándares de comportamiento corporativo (Sartor y Beamish 2019).

Los beneficios para las empresas de participar en el combate al flagelo de la corrupción son numerosos; entre algunos de ellos se encuentran la mejora en su reputación y la generación de adecuadas condiciones de negocio. Al mismo tiempo, las sociedades se tornan más meritocráticas y se fortalece la calidad de la democracia.

En este contexto, donde vienen incrementándose para las pequeñas y medianas empresas (PYMES) las exigencias en materia anticorrupción tanto normativas, como procedentes de los consumidores, resulta oportuno identificar los instrumentos que estas empresas pueden utilizar para evitar caer en conductas inapropiadas, que minen su reputación corporativa y afecten su volumen de negocios. Aquí, se repasarán algunas de las herramientas disponibles cuyos costes económicos cubren un amplio rango.

La estructura del artículo es la siguiente. En un primer momento, se hace un repaso de algunas estrategias anticorrupción disponibles para el sector privado; en un segundo apartado, se habla de herramientas concretas para PYMES; a continuación, se pone especial énfasis en un mecanismo de planeación estratégica, conocido como generación de mapas de riesgo de corrupción. Finalmente, se ofrecen algunas conclusiones en la materia.

\section{SECTOR PRIVADO Y ESTRATEGIAS ANTICORRUPCIÓN}

En los últimos años, se ha extendido la práctica de la creación de departamentos de compliance al interior de las estructuras corporativas. A ello, han conducido presiones de dos tipos. Por un lado, desde el punto 
de vista sociológico, es ingente el número de ciudadanos organizados que a través de diferentes mecanismos exigen altos estándares éticos tanto al sector público como al sector privado. Una herramienta fundamental a tales efectos es el uso de redes sociales. Ante una mínima sospecha de corrupción, la sociedad civil organizada expresa por estas vías su disgusto. A la vez, la responsabilidad social corporativa se ha erigido en una verdadera governance global que no sólo invita a las compañías al respeto de la normativa, sino que la exige (De Colle, Henriques y Sarasvathy 2014). En este plano, el trabajo desarrollado desde Naciones Unidas por Global Compact y sus diferentes redes locales ha sido fundamental.

Por otro lado, la legislación internacional y nacional obliga a la asunción de estos estándares de comportamiento corporativo. A nivel internacional, América Latina fue pionera en el decenio de 1990 con la creación de la Convención Interamericana contra la corrupción. A posteriori, la Convención para combatir el cohecho de servidores públicos extranjeros avanzó pasos en este terreno. Tiempo después, la rúbrica de la Convención de las Naciones Unidas contra la Delincuencia Organizada Transnacional arrojó más luz sobre los criterios a considerar para la detección de comportamientos corruptos. Aunque con sus limitaciones, la "cereza de la tarta" en este proceso ha sido la rúbrica de la Convención de las Naciones Unidas contra la Corrupción.

Desde el punto de vista de la legislación a nivel nacional, dos son los casos más representativos de este cambio de paradigma. Inicialmente, la normativa más potente en la materia fue la Foreign Corrupt Practices Act (FCPA) de 1977 en los Estados Unidos (Carr y Outhwaite 2009, Feathers 2014). Aunque poco aplicada luego de su nacimiento, en los últimos años ha sido la causante de las investigaciones sobre los más resonantes casos de corrupción corporativa. En Europa, la ley más relevante en este dominio es la UK Bribery Act de 2011.

¿Con qué instrumentos cuentan las empresas para adaptarse a estos cambios sociológicos y normativos? El abanico de opciones es amplio. La creación de códigos de conducta para funciones y procesos concretos es una condición sine qua non si se quiere trabajar de manera seria en este campo. La aplicación de herramientas de planificación estratégica, al estilo de generación de mapas de riesgos de corrupción, es otra posibilidad. Estos mapas son, en realidad, matrices que condesan la "probabilidad" de la ocurrencia de hechos de corrupción al interior de los procesos organizacionales. Más adelante en el texto, los mismos serán descritos con mayor detalle. 
Otras acciones consisten en la comunicación interna y externa de los detalles de procesos que podrían eventualmente causar reacciones negativas entre los consumidores. Cuanto mayores dosis de transparencia, mayor será la reputación de la compañía. La protección de la figura de los whistleblowers (denunciantes) es fundamental. En esta línea, la instauración de buzones que protejan el anonimato de quienes comunican la ocurrencia de hechos presuntamente delictivos es fundamental. Gracias a estos "héroes corporativos", fue posible conocer los abusos cometidos en el pasado por organizaciones como Enron o Worldcom.

La estrella de estas acciones son las llamadas collective actions. Un subtipo entre éstas es el de los integrity pacts. En su marco, instituciones públicas, privadas, académicas y del Tercer Sector coordinan esfuerzos para denunciar y concienciar sobre prácticas corruptas. La campańa brasilera Jogos Limpos: Dentro e Fora dos Estádios es un ejemplo de iniciativa exitosa en este terreno. Gracias a ella, pudo garantizarse la "limpieza" de un número importante de procesos licitatorios asociados a la organización del Mundial de Futbol 2014 y de las Olimpiadas de Río de Janeiro en 2016.

Figura 1: Beneficios de las estrategias anticorrupción

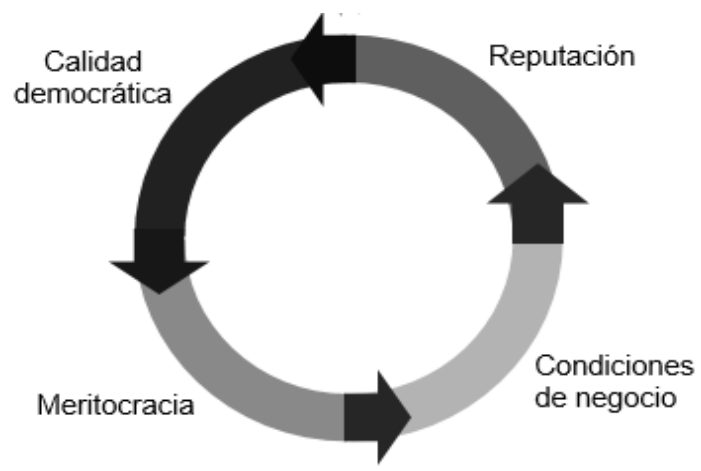

Fuente: Elaboración propia (2019).

La agenda que las empresas tienen por delante en materia anticorrupción es amplia y desafiante. Los resultados de incurrir en acciones de este tipo sólo pueden ser positivos. Las corporaciones mejoran su reputación y generan adecuadas condiciones de negocio. Las sociedades se tornan más meritocráticas porque las "mordidas" ya no son el motivante de la adjudicación de las oportunidades sociales. Los sistemas democráticos, a la vez, ganan en calidad, aspecto no desdeñable en un tiempo histórico 
en el que sectores crecientes de la población se declaran profundamente desafectos (alejados) de las instituciones políticas.

\section{HERRAMIENTAS ANTICORRUPCIÓN EN EL ENTORNO DE LAS PEQUEÑAS Y MEDIANAS EMPRESAS}

Las exigencias tanto normativas como procedentes de los consumidores en materia anticorrupción vienen incrementándose para las PYMES. Afortunadamente, existe un creciente elenco de instrumentos que estas empresas pueden utilizar para evitar caer en conductas inapropiadas que minen su reputación corporativa y afecten su volumen de negocios. Aquí, se repasarán algunas de las herramientas disponibles cuyos costes económicos cubren un amplio rango.

La primera de ellas es el Self Assessment Tool (SAT) que se encuentra disponible online (www.c-detector.eu). El mismo permite identificar áreas de riesgo dentro de procesos diversos de la compañía y, una vez detectados, realiza devoluciones personalizadas con recomendaciones (Italian Union Chambers of Commerce 2014). Los procesos sobre los que diagnostica situaciones vinculadas a corrupción son, entre otros, finanzas y compras, estructura (organigrama), administración y relaciones con el mercado.

También en el campo de la diagnosis se encuentran los mapas de riesgos de corrupción que, mediante un ejercicio colaborativo, permiten prever episodios de esta naturaleza y generar controles para prevenirlos, detectarlos y corregirlos.

En el ámbito operativo, existen dos tipos de códigos que contribuyen en el combate a la corrupción. Nos referimos concretamente a códigos de ética y códigos de conducta. Mientras los primeros explicitan los valores sobre los que descansa la actividad comercial, el segundo describe claramente qué comportamientos son penalizados. Paralelamente, se encuentran los mecanismos para la promoción de la denuncia interna (whistleblowing) que siempre se encuentran acompañados por instancias que protegen a los delatores. La incorporación de estrategias de compliance (responsables encargados de velar por la adecuación del proceder empresarial a la legislación nacional e internacional) también es un tipo concreto de acción en campo operativo.

En el entorno de actividades de monitoreo y evaluación, lo fundamental es que los indicadores asociados a controles de riesgos de corrupción se encuentren claramente definidos y haya responsables precisos de su vigilancia. En el campo de la gestión de los recursos humanos, la prédica/ 
narrativa anticorrupción tiene que ser clara en las fases de reclutamiento, política de remuneración, entrenamiento y rotación de personal (Gopinath 2008). Finalmente, en el área de finanzas, debe vigilarse escrupulosamente a quienes podrían activar pagos de facilitación (Gutmann y Lucas 2018). A la vez, la esponsorización debe ser chequeada a efectos de no estar encubriendo entregas no adecuadas de dinero.

Para dar cabida ordenada a este arsenal de instrumentos, existen modelos de gestión que ayudan a las PYMES en la lucha contra este flagelo. Uno de ellos se vincula a las normas ISO 37001 Standard (Anti Bribery). Otro de ellos está asociado al Pacto Global con sus diez principios. Así mismo, existe la normativa ISO 19600 Standard (Compliance). Para una correcta gestión integral, resulta fundamental la puesta en marcha de estrategias de due diligence que permitan reconocer a las PYMES con quienes interactúan en su cadena de valor. La actuación en el marco de collective actions permite diluir los costes que para unidades independientes puede implicar ejecutar estas herramientas de manera solitaria.

La existencia de corrupción mina las probabilidades de crecimiento de una PYME (Hess 2009). Existe sobrada evidencia empírica que lo respalda. Por eso, es importante avanzar en la incorporación de herramientas como las descritas. La disponibilidad de herramientas low cost y en el marco de partenariados (como aquellos brindados por las cámaras de comercio sectoriales o binacionales) es clave para una correcta adecuación de las compañías a este nuevo escenario global. Caso contrario, los riesgos de pérdida de reputación se elevan y ella, como es sabido, es un activo que cuesta décadas construir y cuya destrucción puede acontecer a velocidad de vértigo.

\section{EL USO DE MAPAS DE RIESGOS DE CORRUPCIÓN EN EL SECTOR PRIVADO}

El combate a la corrupción no es tarea exclusiva de las instituciones y servidores gubernamentales. Los actos atentatorios de la ley que desvían recursos del erario público requieren, indefectiblemente, de la participación de ciudadanos y empresas privadas (Goel, Budak y Rajh. 2015). Diferentes iniciativas y legislación a nivel internacional describen el rol que se prevé para las corporaciones en la lucha contra ese flagelo. Ejemplos de elementos incluidos en esta emergente governance global son la United Nations Convention against Corruption (UNCAC), OECD Convention on Combating Bribery of Foreign Public Officials in International Business Transactions y el Global Compact (Heineman y Heimann 2006). 
De manera paralela, diferentes y concretas herramientas anticorrupción han sido ideadas para su puesta en marcha en entornos propios del sector privado. Como se mencionó anteriormente, un instrumento simple, eficaz y versátil consiste en el uso de mapas de riesgos de corrupción. Los mismos no son sino un mecanismo de planificación estratégica que permite prever eventuales hechos de corrupción al interior de las empresas. Su aplicación debe acontecer a nivel de procesos concretos (que suelen coincidir con dependencias u oficinas específicas dentro del organigrama corporativo) tales como tesorería, compras, gestión de recursos humanos o servicios de venta y postventa.

Figura 2: Ventajas derivadas de la aplicación de mapas de riesgos de corrupción

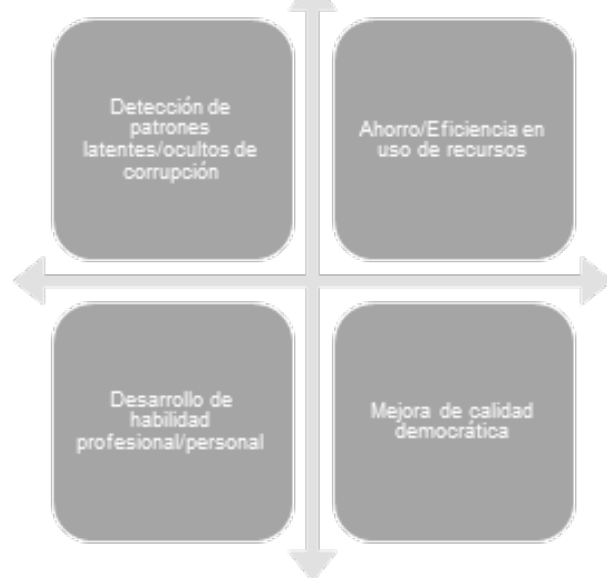

Fuente: Elaboración propia.

A continuación, se enumeran algunos de los beneficios de la aplicación de esta herramienta. En primer lugar, el proceso de identificación de riesgos contribuirá a hacer emerger patrones potenciales de conducta corrupta no previstos anteriormente de manera total o parcial ante circunstancias de este tipo. En segundo lugar, el diseño y puesta en marcha de controles de corrupción permiten un uso eficiente de los recursos. En tercer lugar, la incorporación de la técnica de mapas de riesgos de corrupción implica el desarrollo de una habilidad; esta competencia ayuda a desarrollar todo un modelo de pensamiento y reflexión que es "trasladable" hacia otras esferas a nivel profesional y personal. Y finalmente, a través de vías diferentes, la aplicación de esta herramienta contribuye al incremento de la calidad de la democracia. Por un lado, la detección, control/tratamiento y eliminación de episodios de corrupción contribuyen a revertir los procesos de desafección cívica que alejan a los individuos de la esfera pública y los repliegan en sus vidas personales (Biderbost 2016). 
El diseño de estas matrices informativas corresponde a los líderes y equipos por procesos. Durante el ejercicio ejecutado para conformarlas, se definen causas internas y externas que provocan el riesgo de corrupción. El mismo es definido de manera holística (es decir, identificando quiénes son los responsables y su propósito y determinando donde y cuándo podría tener lugar). A posteriori, para cada riesgo identificado, deben calibrarse probabilidades de ocurrencia e impacto esperado. Esta información constituye los insumos para el diseño de instrumentos que procuren aminorar el riesgo (Biderbost 2016).

Figura 3: Clasificación de los controles

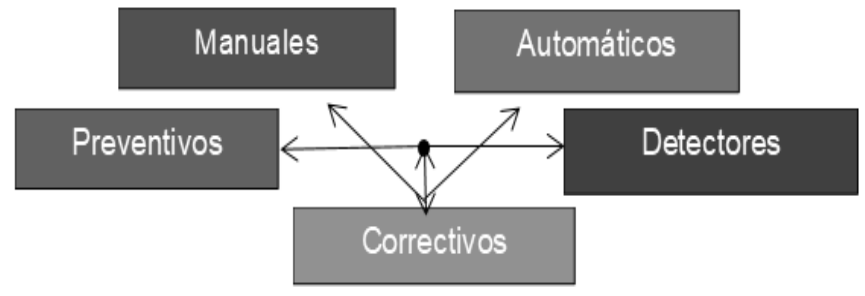

Fuente: Elaboración propia.

Las herramientas que coadyuvan a menguar los niveles de riesgo son denominadas controles. Los mismos son, en sentido estricto, cada una de las acciones que pueden ejecutarse para sublimar los episodios de corrupción. Los controles pueden ser manuales (requieren de la participación de personas) o automáticos (acontecen a partir de lo señalado por softwares o Apps diversas). Se diferencian también en función del momento en el que se activan. Los hay de tipo preventivo, es decir, son aquellos que pretenden evitar el hecho corrupto (como la rotación de personal que forme parte de la apertura de sobres en procesos licitatorios). Existen los de naturaleza detectora o, en otras palabras, los que conforme el acto corrupto tiene lugar lo perciben (como la utilización de programas informáticos que comparen los precios de mercado con los precios ofrecidos por oferentes en un proceso licitatorio). Finalmente, se encuentran los de carácter correctivo. Los últimos son los que se aplican cuando el hecho de corrupción ya ha acaecido (como el uso de sanciones ejemplares a los individuos involucrados).

Una vez definidos riesgos y controles, la matriz construida debe ser compartida con diferentes stakeholders internos (miembros de otros procesos o áreas de la empresa) y externos (medios de comunicación, académicos, sociedad civil organizada, etc.). Es importante remarcar que la ejecución de los controles debe ajustarse a un calendario estricto de actividades cuyo 
cumplimiento debe ser vigilado por instancias de auditoría (designadas ad intra o contratadas ad extra).

Esta herramienta, nacida en el sector privado, viene siendo utilizada incrementalmente también en los sectores de cooperación al desarrollo y en el ámbito gubernamental. Al mismo tiempo, para favorecer su aplicación, se han generado manuales y recursos tecnológicos ad hoc. Entre ellos, se encuentra el web site interactivo que, recientemente y por encargo de UNODC y USAID, hemos diseñado. Esta página web (http://www. mapasderiesgosdecorrupcion.com/), de carácter responsive (accesible a través de ordenadores, tablets y smart phones), permite que los mapas de riesgos de corrupción sean construidos online y, posteriormente, descargados por los interesados. Gracias a este portal web, los tiempos de elaboración y difusión del instrumento se han acortado. Este gadget ha sido incorporado en todas las instituciones del Poder Ejecutivo del Gobierno de Paraguay bajo la coordinación de la Secretaría Nacional Anticorrupción (http:// www.senac.gov.py/) ${ }^{1}$. Además ha sido escogido, por su funcionalidad, por la Veeduría Distrital de Bogotá como una de las diez mejores innovaciones en la materia a nivel mundial ${ }^{2}$. Esta herramienta puede ser útil para otras organizaciones, tanto para desarrollar diagnósticos internos como para mejorar su reputación.

\section{CONCLUSIÓN}

La existencia de corrupción mina las probabilidades de crecimiento de una PYME. Por eso, es importante avanzar en la incorporación de herramientas como las descritas anteriormente. La disponibilidad de herramientas low cost y en el marco de partenariados resultan cruciales para una correcta adecuación de las compañías al nuevo escenario global. Caso contrario, los riesgos de pérdida de un activo tan preciado como la reputación, son elevados.

Aunque la agenda que las empresas tienen por delante en materia anticorrupción es amplia y desafiante, tomar acciones en este sentido sólo puede tener efectos positivos. Como ya se mencionó, las corporaciones

1 Esta herramienta fue generada en el marco del proyecto Consultoría para la Elaboración de la Guía para la Construcción de Mapas de Riesgos de Corrupción (CII UNODC/111/2016). El mismo fue financiado por UNODC, CEAMSO-USAID y Secretaría Nacional Anticorrupción.

2 Más información sobre este reconocimiento se encuentra disponible en el siguiente link: https://bit.ly/2vbcPzM [01-03-2020]. 
mejoran su reputación y generan adecuadas condiciones de negocio. Las sociedades se tornan más meritocráticas y los sistemas democráticos, a la vez, ganan en calidad, aspecto no desdeñable en un tiempo histórico en el que numerosos grupos de la ciudadanía se declaran profundamente desafectos de las instituciones políticas. Finalmente, la lucha contra la corrupción es (y siempre lo ha sido) una tarea compartida. 


\section{REFERENCIAS}

Biderbost, P. (2016). Informe técnico. Guía para la construcción de mapas de riesgos de corrupción. Disponible en http://www. mapasderiesgosdecorrupcion.com/ [1-12-2019].

Carr, I. y Outhwaite, O. (2009). Understanding the impact of anticorruption measures on company practices and attitudes. Agora Without Frontiers: The Journal of the Institute of International Economic Relations, 14 (4), 346-381.

De Colle, S., Henriques, A., y Sarasvathy, S. (2014). The paradox of corporate social responsibility standards. Journal of Business Ethics, 125 (2), 177-191.

Feathers, B. (2014). Bribes without borders: The challenge of fighting corruption in the global context. American University International Law Review, 29 (2), 287-292.

Goel, R. K., Budak, J., y Rajh, E. (2015). Private sector bribery and effectiveness of anti-corruption policies. Applied Economics Letters, 22 (10), 759-766.

Gopinath C. (2008). Recognizing and justifying private corruption. Journal of Business Ethics, 82 (3), 747-754.

Gutmann, J., y Lucas, V. (2018). Private-sector corruption: Measurement and cultural origins. Social Indicators Research, 138 (2), 747-770.

Heineman, B. y Heimann, F. (2006). The long war against corruption. Foreign Affairs, 85 (3), 75.

Hess, D. (2009). Corruption in the value chain: Private-to-private and private-to-public corruption. En Zinnbauer, D., Dobson, R. y Despota, K. (Eds.), Global corruption report: Corruption and the private sector. New York: Cambridge University Press.

Italian Union Chambers of Commerce (2014). Anticorruption toolkit for SMEs (ACTS). Rome: European Commission (HOME / 2014 / ISFP / AG / EFCE).

Sartor, M. A. y Beamish, P. W. (2019). Private sector corruption, public sector corruption and the organisational structure of foreign subsidiaries. Journal of Business Ethics, 1 (2), 1-20.

Recibido: 02-12-2019

Aceptación de la versión final: 30-12-2019 